\title{
REVIEW
}

\section{Reflections on the system of evaluation of gene-edited livestock}

\author{
Ziyao FAN", Tianwen WU', Kui WU, Yulian MU, Kui LI (ه) \\ State Key Laboratory of Animal Nutrition, Institute of Animal Science, Chinese Academy of Agricultural Sciences, Beijing 100193, China
}

\begin{abstract}
The rapid development of biotechnology has provided a greater understanding of the biological functions of major candidate genes that have important functions regarding economic traits, and new materials for livestock breeding have been obtained through gene editing (GE) and embryo manipulation with the purpose of improving quality and output and reducing the costs and risk of disease. Public concerns, particularly over safety risks and production performance, must be addressed. Evaluation is the most important component of the regulation of gene-edited livestock and is a crucial guarantee of public safety before the marketing of geneedited animal products. Here, the system of evaluation of gene-edited livestock is discussed in terms of public safety and production performance. The search for safe and ethical applications in the GE of livestock, a case-by-case evaluation strategy, and classification and simplification are used in order to promote a more efficient, objective, comprehensive and operable evaluation system.
\end{abstract}

Keywords evaluation, gene editing, livestock, performance, safety

\section{From crossbreeding to gene editing (GE)}

Both crossbreeding with the individual animal (the unit of breeding) and genetically modification (GM) breeding with the individual gene (the unit of modification) essentially aim to increase yield and quality, improve animal welfare, reduce costs and eliminate risks during feeding. Animal crossbreeding is based on selection of the complete genetic information carried by individuals or their gametes. Superior individuals or populations are obtained by heterosexual mating and systematic selection. However, barriers such as long generation intervals,

Received December 9, 2019; accepted January 22, 2020

Correspondence: likui@caas.cn

${ }^{*}$ These authors contribute equally to the work reproductive isolation and gene linkage are difficult to overcome to accelerate breeding progress. From 1981, GM was created by the homologous replacement of genes, with the use of vectors, to obtain genetically stable individuals with special abilities ${ }^{[1]}$. GM can both modify a gene for the genetic improvement of a breed or species and also be used for cross-species transfer of genes. More importantly, it can also separate the linkage between advantaged and other genotypes. Certain genes are spatially turned on and off, enhanced and silenced by modifying different components of the genome. The first transgenic livestock were produced in $1985^{[2]}$. However, the huge investment resulted in very low integration efficiency, and embryo microoperation has since limited the development of GM livestock ${ }^{[3]}$. In addition, to increase the probability of obtaining positive cells or embryos, these genetically modified organisms (GMOs) cannot be achieved by the incorporation of donor DNA via the insertion of exogenous screening marker genes into the genome for drug or fluorescence enrichment. This is one of the main areas of public concern ${ }^{[4]}$. More recently, GM livestock have been obtained by homologous recombination (HR) combined with the inducible Cre-loxp element that can eliminate the screening gene, to address public concerns.

GE has been refined and become a new generation GM tool with high efficiency and specificity over the past decade. It mainly comprises zinc finger nuclease (ZFN), transcription activator-like effector nucleases (TALEN), clustered regularly interspaced short palindromic repeat/ Cas9 and structure-guided endonuclease which provide effective new tools for fixed-point modification of animal genomes $^{[5-10]}$. The high recombination efficiency of GE tools can greatly reduce the use of exogenous genes ${ }^{[11]}$.

GE can be defined both narrowly and broadly. As a narrow concept, from the perspective of editing site strategy, it uses ZFN, TALEN, Cas9 and other GE tools. This does not require the introduction of exogenous genes and causes only minimally invasive modifications on the basis of the original genes for creating new structural variation and mimicking single nucleotide polymorphisms 
or indels to achieve changes in the biological function of genes, including whole gene deactivation or quantitative changes in gene expression intensity. This editing strategy has obvious advantages in terms of safety and public acceptance. In this paper the broad concept of GE is discussed from the perspective of technical means which is a relatively efficient gene modification technology. Genomic operations performed by ZFN, TALEN, Cas9 and other GE tools, include gene knockout, inactivation and exogenous gene insertion. Functionally, gene-edited livestock can be classified as livestock generated by GE tools for gene knockout, precise gene modification, gene replacement, site-specific integration, transcriptional activation or post-transcriptional knockout ${ }^{[12]}$.

\section{Concerns regarding gene-edited livestock}

The high efficiency of genome manipulation of the GE tools has greatly reduced the threshold of obtaining geneedited livestock. This provides more genotype for selection combined with somatic cell nuclear transfer, and the use of GE has greatly increased the probability of obtaining positive individuals following microinjection of one-cell embryos. In recent years the species of gene-edited livestock (a new breeding material) have been steadily increasing ${ }^{[13-22]}$. This great genetic progress has also led to social concerns ${ }^{[23]}$. The concerns are mainly about the editing tools and target genes because they are novel.

\subsection{Concerns about GE tools}

A large number of studies have shown that the higher efficiency and specificity of GE tools can lead to reduced potential risks and they more precise than homologous recombination because the probability of random integration can be greatly reduced through specific mediation of guidance nucleic acid. GE is still being refined, especially the Cas9 system which can directly conduct DNA-free GE, and introduce only RNA and Cas9 recombinant protein to achieve non-trace editing and the complete elimination of random integration concerns. The bottleneck due to the refinement needed for off-target prevention and detection remains the main technical problem ${ }^{[24]}$. The Cas 9 system has continuously improved the specificity of GE, including the optimization of the protein structure of Cas 9 and the continuous improvement of sgRNA design rules ${ }^{[25,26]}$. However, the process for the detection of off-target effects requires the prediction of potential off-target sites in the recipient genome through the guidance sequence, and then verification by PCR and DNA sequencing. In addition, next generation sequencing is used to compare the changes in sequence before and after $\mathrm{GE}^{[27-29]}$. However, it is important to note that the mutations found by these processes might not be caused solely by GE, as random mutations in the genome can occur. Also, GE for breeding does not necessarily require all the gene-edited clones to be correct, as the precisely edited individuals or chromosomes can be identified and selected. Recent improvements have helped to prevent off-target effects. Random integration by modified Cas9, reverse transcriptase fusion proteins and a sequence of RNA including sgRNA and RNA sequences are used for reverse transcription into donor $\mathrm{DNA}^{[30]}$.

\subsection{Concerns about the edited gene}

The starting point of GE is to break the existing balance and precisely build a new one which should be closer to the breeding goal than the original one. The biological properties of the edited genes determine the new balance of the individual. Modification of different target genes will lead to deviations in different directions causing retarded development or imbalanced body homeostasis that leads to physiologic defects or premature death. However, the advantages of GE must be emphasized but the potential disadvantages cannot be ignored. The relationship between the threshold of homeostasis of individuals and population in the new balance and nature and society should be comprehensively evaluated. Only the comprehensive evaluation of gene-edited animals will identify the potential commercial value of these livestock and eliminate public concerns over their use. These include pathology and welfare, inputs and outputs, emissions and the environment. However, the evaluation can be simplified and focused based on known gene function. This includes the effect of gene inactivation which should also be evaluated in individuals with biological functions inactivated by mutations caused by GE. In contrast to other GMOs, GE has only so far been applied to mammals, which are more closely related and similar in physiology to humans, so that the negative effects of GE should be prioritized and tested in physiologic systems of mammalian livestock. In addition, mammalian livestock are relatively large compared with other GMOs (e.g., pollen, plant seeds, insects and fish) making the GE product easier to visualize and contain.

\section{Comprehensive evaluation of gene-edited livestock}

The evaluation of gene-edited livestock should reflect favorable and non-harmful ethical principles to ensure both excellent performance and the interests of all stakeholders. Compared with wild types, gene-edited livestock have distinct characteristics in a particular trait such as disease resistance, increased yield, quality, sustainability or fertility (Fig. 1). However, livestock should reflect the 'buckets effect', and whether they can be used 


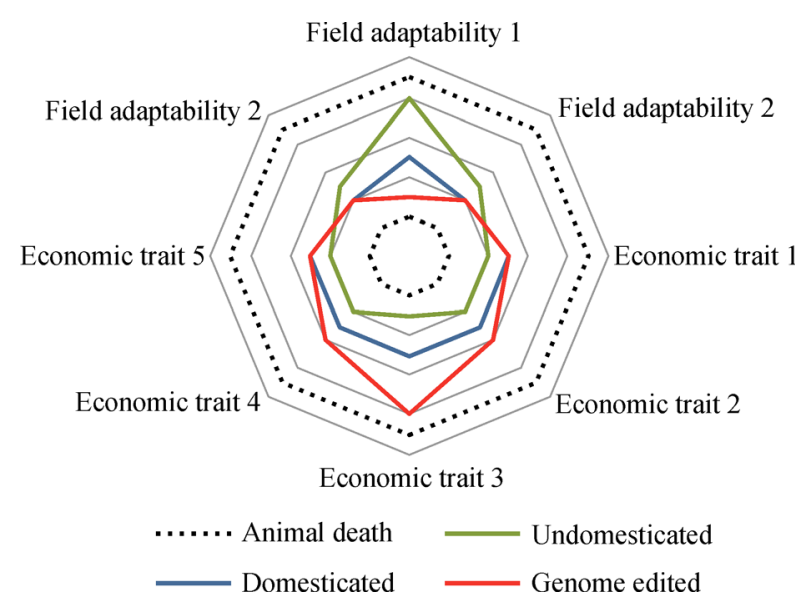

Fig. 1 Schematic diagram of the relationship between development of livestock breeding methods and livestock phenotypes. The area between the dotted lines in the spider web diagram is the range in which individual livestock can survive.

commercially depends on both the improvement of certain economic traits and also the implications of the changes in animal health, physiology, welfare and behavior generated by the GE process $^{[31]}$.

\subsection{Production performance evaluation}

Measurement of production performance is comprehensive and more targeted. For different target genes the case-bycase evaluation strategy should be used, making full use of this species and other higher animal models in which genes related to biological function have been identified. Economic characters, phenotypic relationships and the structure of livestock population levels which verify the variation in the relationship between genotype and phenotype are explored through more targeted analysis.

\subsubsection{Functional efficiency}

Experimental data on the effectiveness of target traits in gene-edited animals under normal conditions or specific conditions are needed to determine functional efficiency. In the case of gene-edited animals that provide protein products for humans, test data of production performance such as meat and milk production should be provided.

\subsubsection{Objective, authoritative and transparent evaluation}

Measurement of production performance is the most important component of new materials for breeding by GE and should reflect the authority and objectivity of the evaluation system. Comprehensive evaluation systems should entrust a third-party authority for measurement of livestock production performance, including feed cost, reproduction, growth and production. The number of individuals assessed should vary with different species and the assessment should report all GE breeding material production performance data, not only to show the advantage of the gene-edited phenotypic but also to reveal other important production performance data. The GE livestock used as new breeding materials should not set any artificial weight of trait indicator, and the right to choose should be given to all stakeholders including consumers.

\subsection{Evaluation of the safety of gene-edited livestock}

Different countries have different understandings of geneedited animals and products and this will lead to potential trade barriers for gene-edited animals and products. We welcome the ability of the government to make some improvements based on our ideas. There is still no broad international consensus on the definition and management of gene-edited livestock ${ }^{[32]}$. The European Court of Justice ruled that gene edited animals must be regulated as GMOs and a previous non-GMO ruling in New Zealand for geneedited animals was struck down by this court. However, in some countries (Argentina, Brazil, Chile, Colombia, Honduras, Japan, Norway, Paraguay and the Philippines) gene-edited livestock are considered as not having foreign DNA insertions (i.e., non-GMO). Livestock with foreign DNA insertions are generally regulated as GMO and follow a case-by-case approach. However, the attitude and approach toward gene-edited livestock remains cautious and uncertain in Australia, China and United States .

Gene-edited livestock should be classified for evaluation and management. The principles of environmental, social, human and animal evaluation, and practicality should be taken as the basic principles of animal evaluation of agricultural GE. Safety evaluation should be based on food safety, feeding safety and ecological safety for the purposes of risk assessment.

\subsubsection{Application scope and classification}

Evaluation needs to be applied to gene-edited livestock and their products obtained through targeted modification of specific sites in the genome by sequence specific nucleases for agricultural production or processing of agricultural products. More GE strategies have been emerging, and the existing classification methods can no longer effectively cover the classification of gene-edited animals, thus we promote a new and more comprehensive classification method to increase work efficiency. GE can be placed into one of seven main categories (Fig. 2):

GE1 Mimicking beneficial natural mutations in breeds of the same species.

GE2 A breed with a gene dysfunction is exist. GE creates a mutation lead to no byproduct of this locus during transcription or translation. 
(a)

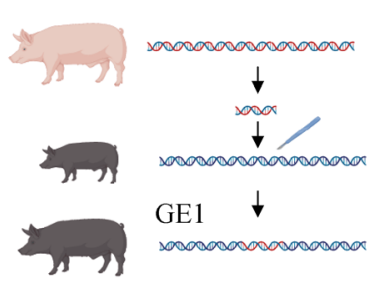

(b)

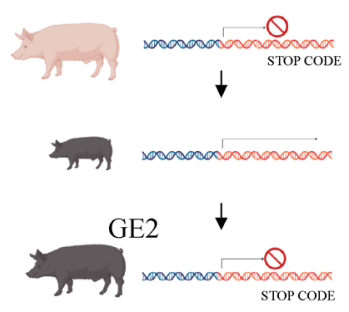

(c)

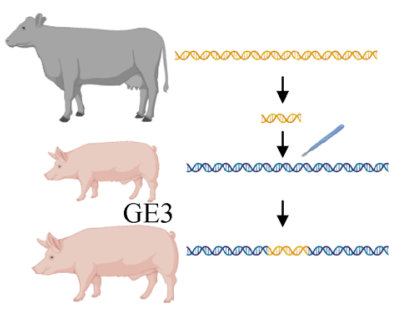

(d)

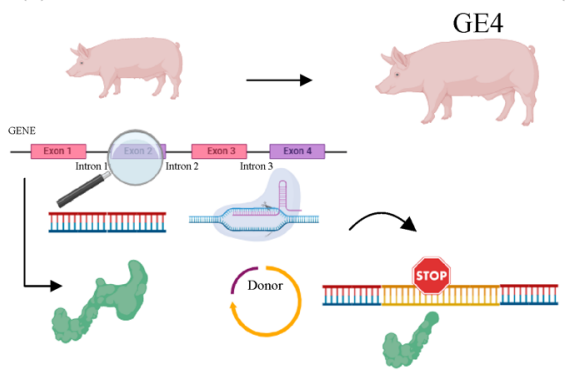

(f)

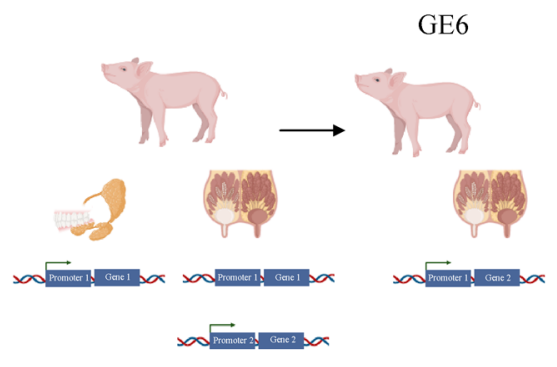

(e)

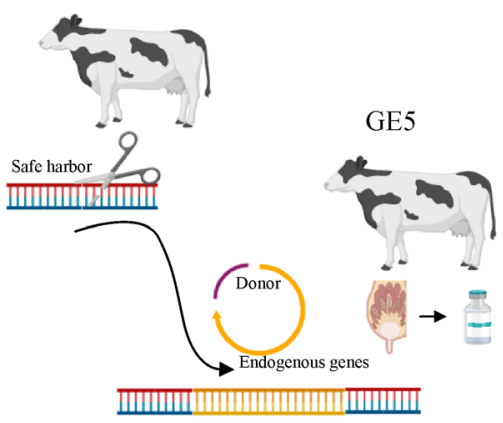

(g)

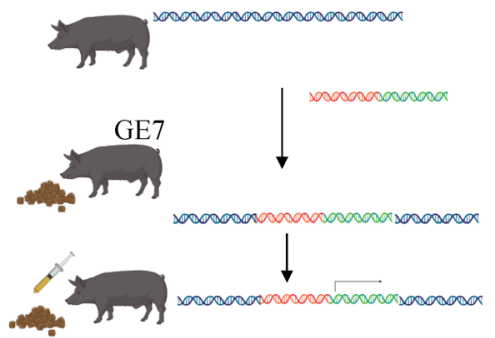

Fig. 2 Schematic diagram of gene-edited livestock classification. (a-g) GE1-GE7.

GE3 Mimicking beneficial mutations or genotypes across species, for example, introducing a naturally occurring bovine mutation into the porcine genome.

GE4 GE made a novel artificial mutation which produced a byproduct of gene during transcription or translation.

GE5 The exogenous gene or regulatory elements are integrated specifically into the genome of livestock by GE tools.

GE6 The spatiotemporal expression of some endogenous genes is changed by reassociation of endogenous genes and endogenous promoters. For example, a geneedited livestock can express an antimicrobial peptide in the breast which is expressed in the saliva in the wild types and not in the breast.

GE7 For inducible gene-edited livestock, inducible conditions include drugs and other means. It is necessary to evaluate the safety of individuals before and after induction and the induction conditions used (e.g., the inductive agent used).

\subsubsection{Management}

\section{Principles}

Management needs to adhere to science-based principles. The safety evaluation of gene-edited livestock needs to be established on the basis of science and objectivity. Case analysis principles are to be used. The safety of geneedited livestock for agriculture varies with receptors, GE methods, approaches and application environments. Risk assessment therefore needs to be conducted according to the specific case and adhere to the principle of classified management. On the basis of risk, different management methods are adopted for different categories of gene-edited organisms used for agriculture. Simplified management methods are adopted for those with low risk, and strict management methods are adopted for those with high risk.

\section{Management based on classification}

The biosafety certificate for GE can be applied directly for precise GE by GE1 and GE2 after it is clear that there is no random integration and there are no off-target effects of the 
GE process. It can be directly used commercially after identifying the genetic stability and suitable breeding performance.

Given that the following contain exogenous genotypes or uncertain functional genotypes, mutations by GE3 to GE7 are required to complete the whole process of experimental research and a pilot test phase by following the rules for agricultural GMO safety evaluation. At the end of the intermediate test phase, management procedures must be determined based on the results of risk assessment and safety assessment. Management can be simplified if the potential risk is small and safety is high. From a technical and ethical perspective, a simplified safety evaluation committee should be established for applications for the decision for simplification which is based on gene function and potential risks in the breeding process. Application can be made for a safety certificate after the completion of a pilot test. If safety cannot be guaranteed, the safety evaluation must be conducted step-by-step in strict accordance with the provisions of the current management measures for safety evaluation of agricultural GMOs.

\subsubsection{Safety evaluation}

To determine if GE3 to GE7 can simplify their management the safety board in the risk assessment and safety assessment will consider whether there are (1) any unexpected gene insertions and editing, (2) new environmental risks, (3) new food safety risks, and (4) opportunities for specific detection methods. Obtaining a safety certificate requires meeting the following conditions:

(1) Molecular characteristics and genetic stability

There is a requirement for provision of PCR data for GE biological identification and tracking; whole genome sequencing and Southern hybridization or other data to prove the insertion sequence of extraneous DNA or transformation vector skeleton residual and unexpected off-target effects; and data on changes in protein size and variables caused by GE.

This part is the basis of the whole evaluation. For example, a recent report claimed that one of the two edited chromosomes of the GE dehorned cattle reported in 2016 was inserted into the sequence of the target plasmid ${ }^{[21,33]}$. Technically speaking, this random integration problem could be detected by NGS and molecular hybridization before the market. Of course, these edited cattle are not useless in animal breeding because another homologous chromosome has been found to be defective and this can be remedied by hybridization with a wild-type individual.

More accurate information about the whole genome should be indicated in order to assess the value of breeding utilization and to guide breeding. Such off-target effects and random integration effects remain a challenge for existing detection methods. It is different to determine whether small structural variations result from heterozygosity of the genome itself or the unintended consequences of GE.

The current methods mainly use the founders of the individual or compare the cell genome and GE before, and by editing sites and bases complementary in principle for potential site prediction for targeted sequencing analysis. The above aspects should mainly serve the purposes of testing and increasing the transparency of individual gene backgrounds in GE.

In addition, the detection of genetic stability largely corresponds to genomic instability caused by GE, such as inserting sequences such as Cas9 that can express DNA recombinant enzymes into the genome. The transposon element is inserted. The insertion of these components leads to genomic instability which may increase the instability of the production performance of GE animals and uncertainty regarding the safety of their use.

(2) Ecological sustainability

The environmental sustainability resulting from the effects of gene-edited animals needs to follow the principle of nonproliferation, low emission, and consideration of species and variety diversity. (a) Greenhouse gases (e.g. $\mathrm{CO}_{2}$ and $\mathrm{CH}_{4}$ ) and fecal and urine pollution caused by the daily intake and discharge of GE animals need to be tracked. (b) Large gene-edited livestock are easy to track but the spread of sperm and positive embryos needs to be controlled. Contingency plans need to be made for the escape and spread of GE animals due to disasters and accidents. (c) The impact on the original species and genetic diversity needs to be considered and the survival, competitiveness and adaptability of GE animals need to be tested. The focus needs to be on the balance between conservation and improvement, to highlight the improvement of planning. (d) If the character of genes has nothing to do with the enhancement of survival competitiveness, the environmental safety assessment may be reduced according to the principle of case analysis, and the reasons for reduction can be provided.

\section{(3) Food safety}

Relevant data on gene-edited livestock in agriculture expressing new peptide chains or proteins need to be provided for toxicological evaluation and sensitization evaluation of the newly expressed substances. Analysis and evaluation of food safety and the impact of production and processing on safety caused by changes in biological components and resulting changes in enzyme activity must also be undertaken.

The food safety of agricultural gene-edited animals needs to be simplified compared with the evaluation standards for transgenic safety. The food safety of GE animals needs to be reasonably simplified but the sensitization effect of nutritional composition analysis and raw and cooked meat states need to be increased as appropriate according to the classification evaluation principle. Safety evaluation of the accumulation of 
potentially toxic metals and drug residues, and of harmful protein products and small biological molecules caused by defective metabolism in vivo must be undertaken. In addition, it is important that an evaluation scheme also adheres to the gene-edited livestock traceability system and information disclosure system.

(4) Feeding safety

(a) Feeder and facility safety

A scoring system has been established for the evaluation of behavior, reaction, stress, mental state and degree of taming in gene-edited livestock.

(b) Feeding adaptability

The ability of gene-edited livestock to survive under the conditions of the standard breeding traits needs to be evaluated to include survival, life span, growth (e.g., birthweight, adult weight, daily gain and growth rate), reproduction (e.g., the estrous cycle, pregnancy rate, semen quality, survival rate of piglets and litter size), disease resistance and susceptibility (infection rate and mortality rate), and adaptability to environmental and physical factors such as temperature and humidity. In addition, suitable specific indicators need to be determined according to the characteristics of the gene-edited livestock.

\section{Summary}

The value of GE in the precise and efficient genetic improvement of livestock is indisputable, especially in terms of increasing yield and quality, reducing costs and disease risk, and increasing animal welfare and environmental friendliness. Systematic and targeted performance measurement and safety evaluation, simplifying the process, scientific classification and reasonable reduction of the evaluation work, are important in the delivery of the benefits of GE. These will help scientists to refine their techniques and select suitable genes to modify and also help the general public to better understand and accept the benefits of livestock GE.

Acknowledgements This work was supported by the National Transgenic Project (2016ZX08006-001), the China Postdoctoral Science Foundation (2018M631648), and the Central Public Interest Scientific Institution Basal Research Fund (2018-YWF-YTS-13).

Compliance with ethics guidelines Ziyao Fan, Tianwen Wu, Kui Xu, Yulian $\mathrm{Mu}$, and Kui Li declare that they have no conflicts of interest or financial conflicts to disclose.

This article is a review and does not contain any studies with human or animal subjects performed by any of the authors.

\section{References}

1. Costantini F, Lacy E. Introduction of a rabbit $\beta$-globin gene into the mouse germ line. Nature, 1981, 294(5836): 92-94
2. Hammer R E, Pursel V G, Rexroad C E Jr, Wall R J, Bolt D J, Ebert K M, Palmiter R D, Brinster R L. Production of transgenic rabbits, sheep and pigs by microinjection. Nature, 1985, 315(6021): 680683

3. Maga E A, Geoffrey Sargent R, Zeng H, Pati S, Zarling D A, Oppenheim S M, Collette N M B, Moyer A L, Conrad-Brink J S, Rowe J D, BonDurant R H, Anderson G B, Murray J D. Increased efficiency of transgenic livestock production. Transgenic Research, 2003, 12(4): 485-496

4. Carlson D F, Tan W, Lillico S G, Stverakova D, Proudfoot C, Christian M, Voytas D F, Long C R, Whitelaw C B, Fahrenkrug S C. Efficient TALEN-mediated gene knockout in livestock. Proceedings of the National Academy of Sciences of the United States of America, 2012, 109(43): 17382-17387

5. Bibikova M, Beumer K, Trautman J K, Carroll D. Enhancing gene targeting with designed zinc finger nucleases. Science, 2003, 300 (5620): 764

6. Miller J C, Holmes M C, Wang J, Guschin D Y, Lee Y L, Rupniewski I, Beausejour C M, Waite A J, Wang N S, Kim K A, Gregory P D, Pabo C O, Rebar E J. An improved zinc-finger nuclease architecture for highly specific genome editing. Nature Biotechnology, 2007, 25(7): 778-785

7. Wood A J, Lo T W, Zeitler B, Pickle C S, Ralston E J, Lee A H, Amora R, Miller J C, Leung E, Meng X, Zhang L, Rebar E J, Gregory P D, Urnov F D, Meyer B J. Targeted genome editing across species using ZFNs and TALENs. Science, 2011, 333(6040): 307

8. Jinek M, Chylinski K, Fonfara I, Hauer M, Doudna J A, Charpentier E. A programmable dual-RNA-guided DNA endonuclease in adaptive bacterial immunity. Science, 2012, 337(6096): 816-821

9. Cong L, Ran F A, Cox D, Lin S, Barretto R, Habib N, Hsu P D, Wu X, Jiang W, Marraffini L A, Zhang F. Multiplex genome engineering using CRISPR/Cas systems. Science, 2013, 339 (6121): 819-823

10. Miller J C, Tan S, Qiao G, Barlow K A, Wang J, Xia D F, Meng X, Paschon D E, Leung E, Hinkley S J, Dulay G P, Hua K L, Ankoudinova I, Cost G J, Urnov F D, Zhang H S, Holmes M C, Zhang L, Gregory P D, Rebar E J. A TALE nuclease architecture for efficient genome editing. Nature Biotechnology, 2011, 29(2): 143148

11. Rouet P, Smih F, Jasin M. Expression of a site-specific endonuclease stimulates homologous recombination in mammalian cells. Proceedings of the National Academy of Sciences of the United States of America, 1994, 91(13): 6064-6068

12. Whyte J J, Zhao J, Wells K D, Samuel M S, Whitworth K M, Walters E M, Laughlin M H, Prather R S. Gene targeting with zinc finger nucleases to produce cloned eGFP knockout pigs. Molecular Reproduction and Development, 2011, 78(1): 2

13. Qian L, Tang M, Yang J, Wang Q, Cai C, Jiang S, Li H, Jiang K, Gao $\mathrm{P}$, Ma D, Chen Y, An X, Li K, Cui W. Targeted mutations in myostatin by zinc-finger nucleases result in double-muscled phenotype in Meishan pigs. Scientific Reports, 2015, 5(1): 14435

14. Yu B, Lu R, Yuan Y, Zhang T, Song S, Qi Z, Shao B, Zhu M, Mi F, Cheng Y. Efficient TALEN-mediated myostatin gene editing in goats. BMC Developmental Biology, 2016, 16(1): 26

15. Wang X, Cai B, Zhou J, Zhu H, Niu Y, Ma B, Yu H, Lei A, Yan H, 
Shen Q, Shi L, Zhao X, Hua J, Huang X, Qu L, Chen Y. Correction: disruption of FGF5 in Cashmere goats using CRISPR/Cas9 results in more secondary hair follicles and longer fibers. PLoS One, 2016, 11(11): e0167322

16. Burkard C, Lillico S G, Reid E, Jackson B, Mileham A J, Ait-Ali T, Whitelaw C B A, Archibald A L. Precision engineering for PRRSV resistance in pigs: macrophages from genome edited pigs lacking CD163 SRCR5 domain are fully resistant to both PRRSV genotypes while maintaining biological function. PLoS Pathogens, 2017, 13 (2): e1006206

17. Gandhi P T, Athmaram T N, Arunkumar G R. Novel nicotine analogues with potential anti-mycobacterial activity. Bioorganic \& Medicinal Chemistry, 2016, 24(8): 1637-1647

18. Wu H, Wang Y, Zhang Y, Yang M, Lv J, Liu J, Zhang Y. TALE nickase-mediated SP110 knockin endows cattle with increased resistance to tuberculosis. Proceedings of the National Academy of Sciences of the United States of America, 2015, 112(13): E1530E1539

19. Yu S, Luo J, Song Z, Ding F, Dai Y, Li N. Highly efficient modification of $\beta$-lactoglobulin $(B L G)$ gene via zinc-finger nucleases in cattle. Cell Research, 2011, 21(11): 1638-1640

20. Zhang J, Cui M L, Nie Y W, Dai B, Li F R, Liu D J, Liang H, Cang M. CRISPR/Cas9-mediated specific integration of fat-1 at the goat MSTN locus. FEBS Journal, 2018, 285(15): 2828-2839

21. Carlson D F, Lancto C A, Zang B, Kim E S, Walton M, Oldeschulte D, Seabury C, Sonstegard T S, Fahrenkrug S C. Production of hornless dairy cattle from genome-edited cell lines. Nature Biotechnology, 2016, 34(5): 479-481

22. Li M, Ouyang H, Yuan H, Li J, Xie Z, Wang K, Yu T, Liu M, Chen $X$, Tang X, Jiao H, Pang D. Site-specific fat-1 knock-in enables significant decrease of n-6PUFAs/n-3PUFAs ratio in pigs. Genetics, 2018, 8(5): 1747-1754

23. Garas L C, Murray J D, Maga E A. Genetically engineered livestock: ethical use for food and medical models. Annual Review of Animal Biosciences, 2015, 3(1): 559-575
24. Zhang X H, Tee L Y, Wang X G, Huang Q S, Yang S H. Off-target effects in CRISPR/Cas9-mediated genome engineering. Molecular Therapy. Nucleic Acids, 2015, 4: e264

25. Fu Y, Sander J D, Reyon D, Cascio V M, Joung J K. Improving CRISPR-Cas nuclease specificity using truncated guide RNAs. Nature Biotechnology, 2014, 32(3): 279-284

26. Zhang J H, Adikaram P, Pandey M, Genis A, Simonds W F. Optimization of genome editing through CRISPR-Cas9 engineering. Bioengineered, 2016, 7(3): 166-174

27. Pattanayak V, Lin S, Guilinger J P, Ma E, Doudna J A, Liu D R. High-throughput profiling of off-target DNA cleavage reveals RNAprogrammed Cas9 nuclease specificity. Nature Biotechnology, 2013, 31(9): 839-843

28. Fu Y, Foden J A, Khayter C, Maeder M L, Reyon D, Joung J K, Sander J D. High-frequency off-target mutagenesis induced by CRISPR-Cas nucleases in human cells. Nature Biotechnology, 2013, 31(9): 822-826

29. Cho S W, Kim S, Kim Y, Kweon J, Kim H S, Bae S, Kim J S. Analysis of off-target effects of CRISPR/Cas-derived RNA-guided endonucleases and nickases. Genome Research, 2014, 24(1): 132141

30. Anzalone A V, Randolph P B, Davis J R, Sousa A A, Koblan L W, Levy J M, Chen P J, Wilson C, Newby G A, Raguram A, Liu D R. Search-and-replace genome editing without double-strand breaks or donor DNA. Nature, 2019, 576(7785): 149-157

31. Maga E A, Murray J D. Welfare applications of genetically engineered animals for use in agriculture. Journal of Animal Science, 2010, 88(4): 1588-1591

32. Duensing N, Sprink T, Parrott W A, Fedorova M, Lema M A, Wolt J D, Bartsch D. Novel features and considerations for ERA and regulation of crops produced by genome editing. Frontiers in Bioengineering and Biotechnology, 2018, 6: 79

33. Norris A L, Lee S S, Greenlees K J, Tadesse D A, Miller M F, Lombardi H A. Template plasmid integration in germline genomeedited cattle. Nature Biotechnology, 2020, 38(2): 163-164 